Ulrich Franke/Ulrich Roos

\title{
Über unerwünschte Handlungsfolgen in den Internationalen Beziehungen
}

\begin{abstract}
Der Beitrag hat die politikwissenschaftliche Teildisziplin der Internationalen Beziehungen selbst zum Gegenstand. Er basiert auf den Annahmen, dass i) im Zuge des realweltlichen Wandels nach dem Ende des Ost-West-Konflikts eine zunehmende theoretische Diversifizierung des Faches erfolgte und ii) dieser Prozess neben neuen Möglichkeiten auch neue Herausforderungen hervorgebracht hat. Als solche Herausforderungen werden unerwünschte indirekte Folgen gegenwärtiger Handlungsroutinen innerhalb der Internationalen Beziehungen verstanden. Sie zu rekonstruieren und mögliche Alternativen vorzuschlagen, ist das Hauptziel des vorliegenden Beitrags.
\end{abstract}

\section{Einleitung - Die Internationalen Beziehungen als Untersuchungsgegenstand}

Viele Jahre habe die politikwissenschaftliche Teildisziplin der Internationalen Beziehungen (IB) zu den westlichen Sozialwissenschaften mit dem geringsten Grad an Selbstreflexion gehört, urteilte Yosef Lapid (1989: 249-250) vor knapp einem Vierteljahrhundert. Und noch vor ein paar Jahren hieß es ebenfalls von prominenter Stelle, die IB-Community solle sich neben dem Weltgeschehen stets auch kritisch »mit sich selbst« auseinandersetzen (Deitelhoff/Wolf 2009: 470). Der vorliegende Artike ${ }^{1}$ kommt dieser Aufforderung nach und lenkt den Blick auf die Binnenstruktur der IB. Ausgehend von der Überlegung, dass die These vom »doppelte[n] Strukturwandel eines Gegenstandes und seines Faches« (Albert 2003) äußerst plausibel ist, stehen dabei jedoch nicht so sehr die Fragen einer teildisziplinären Standortbestimmung oder das Nachzeichnen der konzeptionellen Entwicklung im Vordergrund (vgl. Rittberger/Hummel 1990; Wolf/Hellmann 2003; Deitelhoff/Wolf 2009). Vielmehr geht es um die Frage, welche Regeln das kollektive Handeln in den IB anleiten und welche Wirkungen es zeitigt. Ein solcher Ansatz bietet nicht nur die Möglichkeit, einen zentralen Ausschnitt der Realität des Faches unter die Lupe zu nehmen, sondern er gestattet auch, diese Realität kritisch zu reflektieren und unerwünschte Nebenfolgen des kollektiven Handelns in den IB aufzuzeigen.

Da menschliches Handeln nicht nur beabsichtigte, sondern meist auch unbeabsichtigte Folgen nach sich zieht (die den Beteiligten häufig gar nicht zu Bewusst-

1 Eine frühere Version dieses Beitrags wurde auf dem DVPW-Kongress 2009 in Kiel präsentiert und diskutiert. Unser Dank gilt allen Teilnehmerinnen und Teilnehmern, die durch ihre kritischen Rückfragen, Kommentare und Anregungen zur vorliegenden Fassung beigetragen haben. Ebenfalls danken wir den anonymen GutachterInnen für wertvolle Verbesserungsvorschläge. 
sein kommen), besteht ein Bedarf, auch das Handeln in den IB auf seine nicht-intendierten Folgen hin zu untersuchen. Indem hier also mit den »Spielregeln« und ihren Wirkungen die Binnenstruktur unserer Disziplin ins Zentrum des Reflexionsprozesses gerückt wird, hoffen wir ein Defizit zu verringern, das Christoph Humrich (2006: 73-74) wie folgt beschrieben hat:

»Moreover, if the discipline's members engage in normative critique at all, they publish it in newspapers, more essayistic journals or in popular book series, and thus separate it from the scientific work of the discipline $\ll$.

Gleichzeitig schließen wir an einen wissenschaftssoziologischen Strang der Selbstbetrachtung der akademischen Praxis innerhalb der IB an, der spätestens mit dem Urteil von Ole Wæver zu neuem Leben gelangt ist, dass »the way the discipline usually reflects on its own development falls embarrassingly behind standards developed in sociology of science and historiography « (Wæver 1998: 689). ${ }^{2}$ Auch an den vorliegenden Beitrag ist der Anspruch geknüpft, die von Wæver skizzierte Forschungslücke zu verringern und den Blick auf das Innenleben der IB zu richten. In unserer Wahrnehmung stellen die weltumspannenden Internationalen Beziehungen eine sich fortlaufend verändernde Struktur kollektiven Handelns dar, innerhalb welcher sich zahlreiche nationale oder regionale IB-Kulturen voneinander unterscheiden lassen (vgl. Deitelhoff/Wolf 2009: 452; Tickner/Wæver 2009). Daher beziehen wir uns hier nicht nur auf die IB als global wirkendem Kommunikationsbzw. Kooperationszusammenhang von WissenschaftlerInnen, der sich einer Erforschung der Akteure, Strukturen und Prozesse der Weltpolitik verschrieben hat, sondern - zum Zweck der Illustration - auch und vor allem auf die deutsche IB, die unseren Erfahrungshorizont am stärksten bestimmt.

Im Zentrum unserer Untersuchung steht die Frage, wie sich die Binnenstruktur des Faches Internationale Beziehungen in den vergangenen zwanzig Jahren verändert hat: Welche Handlungsregeln bestimmen über Struktur und Praxis der IB, welche indirekten Handlungsfolgen zeitigen diese Handlungsregeln unter den neuen binnenstrukturellen Bedingungen, und wie lässt sich dies theoretisch-konzeptionell einfangen? Um diesen Fragen nachzugehen, die bislang eher selten Gegenstand der Reflexion geworden sind, orientieren wir uns an den sozialtheoretischen Überle-

2 Auf dieses Urteil reagierten etwa Christian Büger und Frank Gadinger (2007) mit einer von der Wissenschaftsforschung à la Peter Weingart (1972), Karin Knorr-Cetina (1984) und Bruno Latour (2000) inspirierten Untersuchung der Arbeitsbeziehungen zwischen IB-Forschern auf der einen und Politikern, Journalisten, Bürgern, Finanziers oder KollegInnen aus Nachbardisziplinen auf der anderen Seite. 
gungen des amerikanischen Pragmatismus. ${ }^{3}$ Von zentraler Bedeutung sind dabei vor allem als Handlungsregeln gefasste Überzeugungen - die Idee des »belief is a rule for action « (Peirce 1992: 129; vgl. Hellmann 2010) - sowie das Konzept der indirekten Folgen kollektiven Handelns (vgl. Dewey 2001: 37-38). Die Idee der rules for action verweist auf die Annahme, dass das Handeln sozialer Akteure von Handlungsregeln bestimmt wird, die sich im Lauf der Zeit verändern. Bedingt werden solche Veränderungen durch die Erfahrung von Krisenmomenten, in denen sich die bisher befolgten Handlungsregeln als problematisch erweisen und Modifikationen erforderlich machen. Da soziales Handeln - die individuelle oder kollektive Befolgung von rules for action - oftmals unerwünschte Nebenfolgen zeitigt, verweisen die beiden hier für zentral erachteten pragmatistischen Konzepte Handlungsregel und indirekte Handlungsfolge also unmittelbar aufeinander.

Innerhalb der IB sind nun im Zuge der realweltlichen Umbrüche und der darauf reagierenden Veränderungen des Faches in den letzten zwei Jahrzehnten nicht nur neue, unerwünschte und möglicherweise teilweise unbekannte indirekte Handlungsfolgen entstanden, sondern auch Möglichkeiten zu deren produktiver Regulierung, zur Formulierung neuer Handlungsregeln also. Entsprechend werden hier nicht nur Handlungsfolgen analysiert, sondern auch erste Vorschläge zu deren Regulierung formuliert. Dies erfolgt ohne Anspruch auf Vollständigkeit. Vielmehr hoffen wir, eine produktive Debatte darüber anzuregen, wie es gelingen kann, sozialwissenschaftliche Wissensbestände noch systematischer in den Dienst der kritischen Selbstbetrachtung des Faches zu stellen, um so immer sachhaltigere Forschung zu ermöglichen.

Eine solche Überlegung wirft freilich die wissenschaftstheoretische Frage auf, welche Qualität die Arbeit der Mitglieder der IB-Community aufweisen sollte. Im Gegensatz etwa zu Imre Lakatos gelten uns nicht die instinktiven Urteile einiger weniger großer Forscher (vgl. Weingart 1972: 24) als Maßstab für wissenschaftlichen Fortschritt und die Rationalität produktiver Problemverschiebungen (vgl. Lakatos 1970). In Anlehnung an den Pragmatismus sehen wir stattdessen solche Forschungsleistungen als rational und produktiv an, die einen Beitrag zur Lösung der Probleme der Menschheit darstellen; denn für John Dewey (2008: 574) verweisen Probleme der Sozialforschung immer auf gesellschaftliche Spannungen, Bedürfnisse und Probleme. Entsprechend stellt das Lösen von Problemen der Menschheit auch unsere Antwort auf die von Alexander Wendt aus guten Gründen aufgeworfe-

3 Der klassische amerikanische Pragmatismus wurde insbesondere von Charles Sanders Peirce, William James, George Herbert Mead und John Dewey geprägt. Als Neopragmatisten werden unter anderem so verschiedene Denker wie Richard Bernstein, Hilary Putnam und Richard Rorty sowie mitunter auch Jürgen Habermas bezeichnet. In den Internationalen Beziehungen nimmt die Zahl jener, die sich von (neo-)pragmatistischen Ideen beeinflussen lassen, immer weiter zu (vgl. Kratochwil 2009; Friedrichs/Kratochwil 2009; Katzenstein/Sil 2008). In der deutschen IB-Community hat zuerst Gunther Hellmann (2002) das große Potential dieser philosophisch-sozialwissenschaftlichen Tradition erkannt. 
ne und nach wie vor eher selten beantwortete Frage nach dem Wozu unseres Faches dar (vgl. Wendt 2001; Humrich 2006: 76; Risse 2003: 100). ${ }^{4}$

Nach einer kursorischen Darstellung der realweltlichen Umbrüche der letzten zwei Jahrzehnte und der eng damit verknüpften thematischen Entwicklung der IB (Abschnitt 2) erfolgt eine Interpretation dieser Veränderungen (Abschnitt 3). Dabei werden vor allem die Implikationen des doppelten Strukturwandels für die Akteure und Handlungszusammenhänge des Faches in den Blick genommen. Anschließend werden einige unerwünschte indirekte Folgen jener Handlungsregeln rekonstruiert und kritisch beleuchtet, die in den drei Dimensionen Kohärenz des Wissensbestandes (4.1), Ausmaß an Kooperation (4.2) sowie Sichtbarkeit und Diskussion von Forschungsergebnissen (4.3) wirksam sind. Mit einem kurzen Resümee endet der Beitrag (Abschnitt 5).

\section{Realweltliche Veränderungen seit 1989 und ihre thematischen Auswirkungen auf die Internationalen Beziehungen}

Auch wenn sich realweltliche Prozesse und die theoretisch-konzeptionelle Entwicklung der IB nicht voneinander trennen lassen, so folgt der Gang der Wissenschaft doch in der Regel dem realweltlichen Geschehen. Die letzten zwanzig Jahre wurden dabei maßgeblich vom sogenannten Globalisierungsprozess geprägt, einer Entgrenzung der Ökonomie in (teilweiser) Verbindung mit der weltweiten Verbreitung technologischer, aber auch kultureller Errungenschaften. Eng mit der Globalisierung verknüpft sind etwa die Themen Klimawandel, Ressourcenverknappung, Weltbevölkerungszuwachs, transnationaler Terrorismus, die Verbreitung von Massenvernichtungswaffen, Pandemien oder die digitale Revolution. Nicht zuletzt weil sie die Menschheit als Ganze betreffen, dürfte es ein Kennzeichen all dieser Problemfelder sein, dass ihre systematische Erforschung ein höchst drängendes Anliegen darstellt.

Vor diesem Hintergrund identifizierten Klaus Dieter Wolf und Gunther Hellmann (2003: 598) »die gewachsene Distanzierung von staatszentrischen [...] Ansätzen« als »vielleicht wichtigste[s] Merkmal« innerhalb der - deutschsprachigen - IB. Dieser Trend ist ungebrochen und scheint sich allmählich auch auf die IB-Community weltweit auszudehnen - trotz der in den USA nach wie vor dominanten Stellung von Außenpolitikanalyse und IB-Großtheorien. So heißt es etwa bei Robert O. Keohane (2008: 708, Fn. 1):

4 In diesem Zusammenhang wäre es ein Missverständnis zu dem Schluss zu gelangen, die AutorInnen von Beiträgen wie dem vorliegenden verfingen sich in einem performativen Selbstwiderspruch, weil sie sich an der von ihnen geforderten Erforschung der Welt gar nicht beteiligten. Eine solche Interpretation schlösse nicht nur unsere eigene Disziplin aus der »Empirie« aus (denn auch die IB sind ja ein Teil der zu erforschenden Lebenspraxis); mehr noch veranschaulichte sie gerade die Notwendigkeit einer Institutionalisierung der Selbstbetrachtung der IB (in Form einer Sektion Supervision, zum Beispiel) um all jene, die, wie das hier geschehen ist, den Blick auf das eigene Fach richten, von etwaigen Vorwürfen der »Nestbeschmutzung« zu befreien. 
»I use the phrase >world politics $<$ rather than >international relations $<$, since the language of international relations < leads us to think only about states, which are not central to all interesting questions of world politics«.

Darüber hinaus mehren sich in jüngerer Zeit die Stimmen jener, die sowohl das Verschwimmen klarer Grenzen von Innen und Außen beschreiben, als auch das staatliche Gewaltmonopol entlang der Begriffe Intervention und Integration zunehmend kritisch hinterfragen und so zum Beispiel die Zukunft staatlicher Außenpolitik insgesamt äußerst trübe zeichnen. ${ }^{5}$ Insbesondere die in den letzten fünfzehn Jahren immer stärkeren Zulauf erhaltende global governance-Forschung hinterfragt die zukünftige Relevanz einzelner Staaten und deren Außenpolitiken sowie die Idee des Nationalstaats und die Bedeutung territorialer Grenzen. Das um sich greifende Desinteresse an Außenpolitikforschung, wie es Walter Carlsnaes (2002: 331) diagnostiziert, geht mit dem Erstarken der global governance-Modelle Hand in Hand. Letztere billigen den Nationalstaaten und deren Außenpolitiken eine immer geringere Relevanz für die Dynamiken des Weltgeschehens zu. So überrascht nicht, dass das Interesse an staatszentrierter Forschung dort nachlassen muss, wo beispielsweise die Erosion des nationalstaatlichen zugunsten der Emergenz transnationalen Regierens angenommen (vgl. Joerges 2001; 2006) beziehungsweise die Entwicklung von der »nationalen zur post-nationalen Konstellation« beschrieben wird (vgl. Zürn 2005; Zangl/Zürn 2003; Leibfried/Zürn 2006). ${ }^{6}$ Diesen theoretischen Überlegungen ist gemeinsam, dass sie die Bedeutungsverluste und -gewinne bestimmter Akteure oder Institutionen aus einer Nullsummenspiellogik heraus begreifen (vgl. Sending/ Neumann 2006: 651; Ruggie 2004: 519). Das heißt, wer die Welt aus dieser Perspektive betrachtet und zu der Auffassung gelangt, dass verschiedene nicht-staatliche Akteure immer mehr Einfluss in der Weltpolitik gewinnen, wird im gleichen Maße einen Machtverlust von Nationalstaaten und deren Außenpolitik unterstellen (vgl. Keck/Sikkink 1998).

So nimmt es nicht wunder, dass der Fokus neuerer Ansätze innerhalb der Disziplin immer stärker vom Nationalstaat abgelöst und stattdessen etwa auf als spheres of authority verstandene transnationale Politiknetzwerke gerichtet wird, an denen Staaten zwar beteiligt sind, aber nicht notwendigerweise eine dominante Rolle ein-

5 Besonderer Dank gebührt an dieser Stelle Gunther Hellmann und Benjamin Herborth, die sich die Zeit genommen haben, den Inhalt dieses Abschnitts (in einem anderen Arbeitszusammenhang) kritisch zu kommentieren.

6 Als wichtiger Meilenstein dieser Ansätze gilt die Idee des Regierens »jenseits des Nationalstaats « (Zürn 2005). Internationale Institutionen stellen dort nicht länger die Ergänzungen und Instrumente einer nationalstaatlich bestimmten Welt dar, sondern sind Ausdruck einer politischen Denationalisierung und als solche »Stationen auf dem Weg zu global governance«. Durch zunehmende Verrechtlichung und Vergesellschaftung entsteht in dieser Perspektive eine politische Ordnung jenseits des Nationalstaats, so dass dieser nicht mehr die exklusive Form politischer Herrschaft bildet und nunmehr auch auf transnationaler Ebene über die gute politische Ordnung gestritten wird (vgl. Zürn 2005: 408). Ähnlich gehen Finnemore/Sikkink (1998), Risse/Ropp/Sikkink (1999) und Checkel (2001) davon aus, dass inter- bzw. transnationale Normen die Identitäten der Staaten verändern und deren außenpolitisches »Verhalten« bestimmen. 
nehmen (Rosenau 1999). ${ }^{7}$ Globalisierung laufe zwar außerhalb der Reichweite der Staaten ab, verändere aber gleichwohl deren Identitäten (vgl. Cerny 1995: 596, 1997: 253-273; Sassen 1996; Bartelson 2000: 188). So verstanden gelten Staaten und deren Außenpolitiken nur noch als Vollstrecker von Kräften, die sie nicht länger zu kontrollieren vermögen. In dieser Perspektive erscheint Globalisierung als ein von seiner eigenen Dynamik angetriebener Prozess (vgl. Bartelson 2000: 189). ${ }^{8}$

Auf diesen allgemeinen Trend zur Aufweichung des Staatszentrismus in den neuen Internationalen Beziehungen antworten die alten Internationalen Beziehungen mit der Verfeinerung ihrer Theoriemodelle. So reagieren die Protagonisten der Außenpolitikanalyse auf den Befund der zunehmenden weltpolitischen Akteursdiversifizierung, indem sie sich für die Analyse nicht-staatlicher Außenpolitiken öffnen (vgl. Smith et al. 2008: 2). Dabei wird der traditionelle Nexus von Nationalstaat und Außenpolitik so weit gelockert, dass nunmehr auch internationale Organisationen, Nicht-Regierungsorganisationen und transnationale Konzerne Außenpolitik betreiben. Ein solches Flechten von Schutzgürteln aus Hilfshypothesen zur Rettung der theoretischen Kernannahmen (vgl. Lakatos 1970) lässt sich nicht zuletzt im neorealistischen Forschungsprogramm feststellen. Dort werden die zentralen Argumente konkurrierender Ansätze zunehmend absorbiert und in Form von intervenierenden Variablen in die teilweise bis zur Unkenntlichkeit modifizierten Theorien integriert (vgl. Crawford 2007; Karp 2009).

Trotz dieser Abwehrmaßnahmen und obwohl das neorealistische Forschungsprogramm gerade in den USA noch immer den Diskurs (mit-)bestimmt, geraten IBTheorien in Anbetracht der kaum zu leugnenden Relevanz nicht-staatlicher Akteure zunehmend in Schwierigkeiten, da sie deren Funktion und Rolle in den internationalen Beziehungen nicht adäquat erklären können, wenn sie die Konsistenz ihrer Prämissen wahren wollen. Ebenso aus der Mode wirken die klassischen Ansätze der Außenpolitikanalyse. Das »Verhalten« oder Handeln eines einzelnen Staates durch die Analyse der Beschaffenheit seines politischen Systems und der Eigenschaften der Regierungsmitglieder erklären zu wollen, erscheint in theoretischer Hinsicht angesichts der immer weiter fortschreitenden Interdependenz und Verflechtung des Weltgeschehens in theoretischer Hinsicht zunehmend unplausibel. Selbst wenn man bereit ist, der Frage, warum sich Staat X in Situation Y auf Art und Weise $\mathrm{Z}$ verhält, trotz des Interdependenzbefundes eine gewisse Relevanz zuzuerkennen, so ist doch evident, dass staatliche Außenpolitik nur noch ein Element

7 Diese Herangehensweise basiert unter anderem auf der Idee, »government « nicht mehr länger als Institution, sondern als Prozess zu konzeptualisieren (vgl. Rosenau/Czempiel 1992), um auf diese Weise den angenommenen Trend weg von hierarchischen, souveränitätsbasierten Modi des (staatlichen) Regierens hin zu stärker horizontalen, netzwerkbasierten (transnationalen) Regierungsformen angemessen analysieren zu können.

8 Jens Bartelson geht unter Rückgriff auf die Idee des »flows« bei Timothy W. Luke (1993: 240) sogar so weit, anzunehmen, dass ein Netzwerk nicht-räumlicher Flüsse die Unterscheidung von System und Einheiten beziehungsweise von internationaler Politik und Außenpolitik hinwegspülen wird. Ähnlich wie Michael Zürn erscheint deshalb auch Bartelson die Auflösung des Staates und des internationalen Systems durch eine Entterritorialisierung der Identitäten sowie deren Entkopplung von politischer Autorität wahrscheinlich (vgl. Bartelson 2000: 190). 
unter vielen ist, die das Weltgeschehen bestimmen. IB-Theorien und Außenpolitikanalyse kranken insofern beide daran, dass die internationalen Beziehungen nur noch eine von vielen Dimensionen der Weltpolitik darstellen.

Vor diesem Hintergrund verstehen wir die global governance-Ansätze als Reaktion auf die Krise der klassischen IB-Theorien. Sie modifizieren deren Explanandum, indem sie die Erklärung weltpolitischer Interaktion an die Stelle des Verhaltens von Staaten im internationalen System rücken - und sie arbeiten mit einem unterschiedlichen Explanans, denn sowohl die Eigenschaften des Systems als auch die Attribute der Akteure werden ontologisch neu gefasst. So nimmt die Systemeigenschaft Anarchie keinen zentralen Rang mehr ein. Stattdessen werden Interdependenz, Kooperation, Kommunikationsflüsse, Weltgesellschaft, der Bedeutungsverlust des Nationalstaats und seiner territorialen Grenzen, Netzwerke, gemeinsame Normen und Identitäten sowie die Verregelung von Interaktion betont. Ebenfalls eine deutliche Steigerung verbucht im Rahmen der global governance-Ansätze die Anzahl der Akteurstypen. Neben die Staaten rücken transnationale Unternehmen und Nichtregierungsorganisationen, Public Private Partnerships, die zivile Weltgesellschaft, Regime sowie inter- und supranationale Organisationen.

Somit spricht immer weniger dafür, dass die Bezeichnung Internationale Beziehungen den theoretischen Prämissen dieser neuen Ansätze gerecht wird und sie angemessen repräsentiert. Und auch wenn eine sofortige Umbenennung der IB mutmaßlich nicht von jedem gewünscht wird, dürfte - getragen von der weiter steigenden Zahl derer, die sich nicht mehr als »IBler«, sondern als Erforscher von Weltpolitik verstehen - eine schleichende Namensänderung im Zuge thematischer Neuausrichtungen bereits in vollem Gange sein. Im Gegensatz zur Bildung einer neuen Teildisziplin neben der klassischen IB, durch die nur unnötige neue Grenzen gezogen würden, bliebe in der mittelfristig wohl von immer weiteren Teilen der Community neu als Weltpolitik bezeichneten Disziplin auch künftig genügend Platz für die klassischen IB-Theorien à la Hedley Bull (1977), Kenneth N. Waltz (1979) und Alexander Wendt (1999) sowie für die Außenpolitikanalyse.

Zusammengefasst ist die hier kursorisch nachgezeichnete Theorieentwicklung eng mit den revolutionären realweltlichen Ereignissen der Jahre nach 1989 verknüpft. So wie seither die Souveränität der Nationalstaaten und damit deren unangefochtene Problemlösungskompetenz in Frage gestellt wird, geraten auch die darauf basierenden Forschungsprogramme in Erklärungsnot. Mit dem Souveränitätsverlust der Nationalstaaten geht also ein Problemlösungskompetenzverlust der klassischen IB-Forschungsprogramme einher (vgl. Griffiths/O'Callaghan 2001: 189), der sich primär in folgenden vier Entwicklungen manifestiert:

- die zentrale Stellung des Staates und des Staatensystems büßen weiterhin tendenziell an Bedeutung ein;

- $\quad$ in Folge dessen gewinnen neue Ansätze zu Lasten der klassischen IB-Theorien und Außenpolitikanalyse-Instrumentarien in der Disziplin an Einfluss;

- die Menge der verschiedenen theoretischen Ansätze nimmt immer weiter zu;

- und die älteren Forschungsprogramme reagieren darauf mit der Formulierung immer neuer Hilfshypothesen. 


\section{Implikationen der realweltlichen und thematischen Veränderungen für Akteure und Struktur der Internationalen Beziehungen}

Die oben kurz skizzierten weltpolitischen und thematischen Veränderungen gehen an der Struktur der IB nicht spurlos vorüber. So gliedert sich das Fach in immer zahlreichere Teil-Teildisziplinen. Ein Blick auf die 24 Abteilungen der International Studies Association (ISA 2011) macht dies schnell deutlich. Die realweltlichen Prozesse erfordern nicht nur eine immer feinere Gliederung der älteren Sektionen unseres Faches, sondern auch die Schaffung gänzlich neuer Forschungsfelder und die systematische Inkorporation von Wissensbeständen aus Nachbardisziplinen.

Was die organisatorische Ausgestaltung der Binnenstruktur des Faches anbelangt, so wurden die vergangenen zwei Jahrzehnte stark von der Gründung überstaatlich-regionaler Fachverbände geprägt. Neben dem im Jahr 2000 gegründeten World International Studies Committee (WISC 2009), dessen Ziel lautet »to make international studies truly universal , sind hier vor allem zu nennen die Standing Group on International Relations des European Consortium for Political Research (gegr. 1991), die Nordic International Studies Association (gegr. 1991), die Central and East European International Studies Association (gegr. 1996) oder die Asian Political and International Studies Association (gegr. 2002). Diese Entwicklung in einen Zusammenhang zu rücken mit der im selben Zeitraum zu beobachtenden Entgrenzung nationaler Räume liegt durchaus nahe. ${ }^{9}$

Die zunehmende Diversifizierung und Globalisierung des Forschungsgegenstands wird also mit einer zunehmenden Diversifizierung und Globalisierung der Disziplin beantwortet. Ein solch umfänglicher Wandel vollzieht sich jedoch nicht ohne unbeabsichtigte oder indirekte Handlungsfolgen. Im Anschluss an Dewey gelten indirekte Folgen kollektiven Handelns als eine universelle Eigenschaft menschlicher Zusammenschlüsse (vgl. Dewey 2001: 37-38). Dabei stellt es eine doppelte Herausforderung dar, Handlungsregeln so zu verändern, dass die gewünschten Handlungsfolgen reproduziert, die unerwünschten aber vermieden werden.

Den oben beschriebenen Strukturwandel des Faches häufiger in den Blick zu nehmen und die damit einhergehenden (teilweise unbekannten) Auswirkungen systematischer zum Gegenstand der Forschung zu machen, erscheint uns vor allem deshalb lohnenswert, da sich das Theoriegefüge der alten Internationalen Beziehungen im Zuge der realweltlichen Umbrüche spürbar gewandelt hat. Neue Ansätze

9 Gleichwohl soll die gegenwärtige Praxis des Faches hier keineswegs allein im Lichte der vergangenen zwei Jahrzehnte verstanden werden. Vielmehr bietet es sich an, bis dato mindestens drei Phasen der Geschichte der IB als universitärer (Teil-)Disziplin zu unterscheiden:

- eine Gründungsphase zwischen dem Ende des Ersten Weltkrieges und dem Höhepunkt der Dekolonisierung;

- eine erste Phase der Ausdifferenzierung und Spezialisierung bis zum Ende des OstWest-Konflikts;

- und eine zweite Phase der Ausdifferenzierung und Spezialisierung seit 1990.

Jede dieser Phasen, dies sei ergänzt, geht einher mit der Gründung diverser Fachzeitschriften von immer weiter steigender Spezialisierung. 
sind entstanden; es herrscht eine neue Vielfalt. ${ }^{10}$ Gleichzeitig ist die Disziplin aber auch unübersichtlicher geworden. Neue Vokabulare sorgen für eine Zunahme der Distanz zwischen den Ansätzen. Dass diese Entwicklung nicht ohne Wirkung auf die Kommunikationsstrukturen der Disziplin blieb, kann nicht verwundern. Wenn aber Verständigung mittels einer gemeinsamen Begriffssprache kaum noch möglich ist, resultiert daraus meist eine gewisse Beschränkung des Austauschs auf diejenigen Kollegen, die ohnehin eine Perspektive miteinander teilen. ${ }^{11}$

Positiv kann diese Entwicklung als Theorienpluralismus bezeichnet werden. Erinnert man sich daran, dass Volker Rittberger und Hartwig Hummel (1990: 20) vor zwanzig Jahren einen »Mangel an theoriegeleiteter oder zumindest theoretisch orientierter Forschung « konstatierten, so wird deutlich, welch' enorme Fortschritte konzeptionell in diesem Bereich erfolgt sind. Kritischer bewertet, lässt sich indes der immer weiter abnehmende common ground beklagen - eine um sich greifende neue Unübersichtlichkeit des Faches. Dass sich in dieser Sorge vor einem Mangel an Kohärenz vor allem technokratische Kontrollphantasien oder Disziplinierungsversuche ausdrücken, wäre dagegen zu einfach. Infolge der wachsenden Distanz zwischen den einzelnen Theorien müssen wir uns immer stärker anstrengen, um die Stimmen der anderen überhaupt noch wahrzunehmen. Während die Teil-Teildisziplinen immer weiter auseinanderdriften, schwindet zusehends das Interesse, welches die alten IB und die jüngeren world politics- oder global governance-Ansätze füreinander aufbringen. Mittelfristig nimmt damit aufgrund einer gewissen sprachlichen Entwöhnung die Fähigkeit ab, sich wechselseitig zu verstehen und die Sprache der anderen in das eigene Bedeutungssystem zu übersetzen.

Wem dies zu abstrakt klingt, der überprüfe einmal kritisch die eigenen Lesegewohnheiten anhand der Fachzeitschriften der International Studies Association: Welcher »Quanti« macht sich denn die Mühe, in der International Political Socio$\operatorname{logy}$ zu lesen - und welcher »Quali« ist noch willens (und in der Lage), die Regres-

10 Prima facie scheint dieser Befund einer neuen Vielfalt im Widerspruch zu alternativen Beschreibungen jüngeren Datums zu stehen, die entweder von einer enorm gestiegenen Kohärenz der Disziplin (vgl. Humrich 2006: 74) oder von einem gemeinsamen »konzeptionellen Dach" ausgehen, unter dem es "zunehmend auch [zu einer] konzertierten Bearbeitung « wichtiger Untersuchungsfelder gekommen sei (Deitelhoff/Wolf 2009: 458). Auf den zweiten Blick wird jedoch deutlich, dass sich diese Interpretationen keineswegs ausschließen. Denn während sich unser Vielfaltsbefund auf den Stand der Disziplin weltweit bezieht, orientiert sich der jeweils unterschiedlich bewertete, aber dennoch geteilte Homogenitätsbefund von Humrich und Deitelhoff/Wolf auf die deutsche IB.

11 Vgl. Mathias Albert (2003: 571), der vor dem Hintergrund einer sehr ähnlichen Diagnose für den Verzicht auf eine Eingrenzung des Gegenstandsbereichs der neuen Internationalen Beziehungen plädiert und eine "gesellschaftstheoretisch reflektierte Orientierung auf weltgesellschaftliche Zusammenhänge« empfiehlt. Während Alberts diesbezügliche Orientierung eher von Niklas Luhmanns Systemtheorie inspiriert ist, wurzelt unsere gesellschaftstheoretische Perspektive, wie oben dargelegt, eher im amerikanischen Pragmatismus. Diese Differenz dürfte auch dann wirksam werden, sollte es sich bei den hier rekonstruierten Entwicklungen nicht um ein Spezifikum der IB, sondern um einen alle Wissenschaften betreffenden Differenzierungs- und Spezialisierungsprozess handeln. Denn während Systemtheoretiker daraus eine gewisse Unabänderbarkeit des Laufs der Dinge abzuleiten geneigt sein könnten, dürften Pragmatisten eher darauf beharren, sich diesem mit ihren Überzeugungen entgegenzustellen. 
sionsanalysen in International Studies Quarterly en detail nachzuvollziehen? Angesichts der abnehmenden Bedeutung quantifizierender Arbeiten in den IB mag dieses Beispiel heute - zumindest aus deutscher oder europäischer Sicht - vielleicht weniger Geltung beanspruchen können, als dies noch vor ein, zwei Jahrzehnten der Fall gewesen ist; worauf es uns hier ankommt, ist jedoch Folgendes: Während die einen darauf hoffen, dass die neuen Theoriegebäude schon bald wieder einstürzen, sind die anderen davon überzeugt, dass es die alten Ansätze sind, die sich in Kürze vollständig erledigt haben werden. Die Quintessenz der Haltung beider Gruppen ist indes ungefähr gleich. Zugespitzt formuliert lautet sie: Unter keinen Umständen von den Annahmen und Methoden der jeweils anderen irritieren oder gar aufhalten lassen. Dass sich Einzelne noch immer dafür einsetzen, die unterschiedlichen Ansätze zum gegenseitigen Nutzen zu übersetzen und ihre Anhänger einander wieder näher zu bringen, steht dem nicht entgegen. Der Anteil jener aber, die ihre Aufmerksamkeit den Arbeiten anderer (Sektionen, Theorien, usw.) versagen, nimmt wohl stetig zu.

\section{Rekonstruktion unerwünschter indirekter Handlungsfolgen in den neuen Internationalen Beziehungen}

Auch unser disziplininternes Handeln zeitigt unbeabsichtigte Folgen, die zu regulieren wünschenswert erscheint. Auf Grundlage dieser Annahme soll das verfügbare theoretische Wissen über indirekte Folgen kollektiven Handelns nun reflexiv auf jene Veränderungen angewendet werden, von denen die Strukturen unseres eigenen Faches in den beiden letzten Jahrzehnten erfasst worden sind. Zu diesem Zweck werden wir einige der als indirekte Handlungsfolgen verstandenen Wirkungen des doppelten Strukturwandels von Fach und Gegenstand rekonstruieren - und Möglichkeiten zu deren Regulierung vorschlagen. Dies erfolgt anhand von drei zentralen Dimensionen des kollektiven Handelns in den IB: der Kohärenz des Wissensbestandes, dem Ausmaß an Kooperation und der Sichtbarkeit und Diskussion von Forschungsergebnissen. Einen Anspruch auf Vollständigkeit erheben wir damit nicht. Vielmehr laden wir zur Reflexion darüber ein, welche weiteren unerwünschten Folgen das kollektive Handeln in unserer Teildisziplin zeitigt. Die gemeinsame Rekonstruktion bislang verborgener Handlungsregeln, nebst den damit verbundenen unbeabsichtigten Folgen, vermag unser Fach ohne Zweifel wirksamer und relevanter zu machen. Zudem könnte so ein Beitrag zu jenem kühnen Vorhaben geleis- 
tet werden, welches Harry Bauer und Elisabetta Brighi (2009: 165) als »Demokratisierung der IB « bezeichnen. ${ }^{12}$

\subsection{Kohärenz des Wissensbestandes}

Obwohl die Theoriegebäude in den neuen IB aufgrund des dringenden Verdachts errichtet worden sind, dass sich die Produktivität der herkömmlichen Forschungsprogramme erschöpft habe, überblicken heute nur noch die wenigsten Konstruktivisten, Post-Positivisten oder global governance-Forscher die Entwicklung der Ansätze in den alten IB. Dem Alten einmal den Rücken zugewandt, sind sie nunmehr ganz und gar mit sich selbst beschäftigt. Mit Dewey (2004: 145) gesprochen:

»Die Menschen verlieren das Interesse an Dingen, die für sie früher von größter intellektueller Bedeutsamkeit waren; Ideen verlieren ihren Glanz; ehemals dringliche Anliegen erscheinen fern. Die Menschen blicken in eine andere Richtung; was sie früher in Verwirrung gestürzt hat, ist unwirklich geworden; Erwägungen, die als unerheblich übergangen wurden, treten in den Vordergrund. Frühere Probleme sind vielleicht nicht gelöst worden, verlangen aber auch nicht weiter nach einer Lösung«.

Dewey legt hier den Gedanken nahe, dass sich der Fokus des wissenschaftlichen Interesses verlagert, wenn sich die Wahrnehmung von Wirklichkeit verändert. Im Unterschied zu der Idee sprunghafter wissenschaftlicher Revolutionen bei Thomas Kuhn (1976) zeichnet er also das Bild einer sich fortlaufend wandelnden Wissenschaft. Daraus leitet sich das Erfordernis ab, während der einzelnen Phasen dieser kontinuierlichen Entwicklung eine niemals abreißende Selbstvergewisserungsdebatte darüber zu führen, welche Art von Beiträgen gerade als besonders nützlich und fruchtbar gelten sollte. Wenn zudem die oben angestellten Überlegungen zur innerhalb der Community abnehmenden Übersicht über die Theorien, Fragestellungen, Methoden und Befunde der einzelnen Sektionen als plausibel gelten können und wenn ein verbesserter Überblick zu (mehr) fruchtbringender Kooperation befähigt und Redundanzen zu vermeiden hilft, dann sollten wir die Anerkennungsstruk-

12 Deitelhoff und Wolf rekonstruieren in ihrem Beitrag ebenfalls zwei indirekte Folgen der etablierten Handlungsregeln unseres Faches. So drohe die deutsche IB-Community ihren eigenen Kommunikationsraum (etwa die Zeitschrift für Internationale Beziehungen, ZIB) zu verspielen, indem sie sich auf das Credo Publiziert auf Englisch einlasse. Als indirekte Handlungsfolge würde die ehedem originelle deutsche Argumentationskultur so »häufig den gleichen sterilen, soliden, aber mehr oder weniger pointenfreien Charakter« der USamerikanischen Forschung annehmen. Die dort platzierten Ergebnisse würden »jedenfalls keine spannenden Debatten mehr« entzünden und »könnten so gerade die Innovationskraft der deutschen IB hemmen«. Im Blick auf die »auf uns zukommenden bibliometrischen Kriterien für die Leistungsbemessung von Forschung« verweisen Deitelhoff/Wolf darauf, dass einige Kollegen keine Bücher mehr schrieben, »weil ein Aufsatz in einem top journal soviel zählt wie eine Monografie, und andere publizieren eben nicht mehr in der ZIB, weil sie nicht im Social-Science-Citation-Index berücksichtigt ist«. Die indirekte Handlungsfolge wird dabei in Frageform präsentiert: »Geht auf diesem Weg genau das verloren, was in den vergangenen Bestandsaufnahmen so positiv gewertet wurde, die Originalität und die intradisziplinäre Aufgeschlossenheit und Pluralität? « (alle Zitate: Deitelhoff/Wolf 2009: 469, Hervorhebungen im Original). 
tur innerhalb unseres Faches entsprechend modifizieren. Stärker gefördert und mit mehr Anerkennung versehen werden müssten in der gegenwärtigen Phase der Entwicklung unserer Disziplin also Überblicksbeiträge, die den Forschungsstand einer Sektion komprimiert und auf höchstem Niveau darstellen oder die Wissensbestände verschiedener Sektionen im Blick auf eine sie verbindende Fragestellung in kreativer Weise neu anzuordnen helfen. Solange jedoch theoretische Innovationen am stärksten prämiert werden und damit den Weg zur erstrebten Anerkennung am wahrscheinlichsten machen, wird der Mangel an Überblicksarbeiten weiterhin Bestand haben. ${ }^{13}$

Dass überhaupt ein Defizit an Überblicksarbeiten besteht, halten wir für eine indirekte Handlungsfolge, die sich aus der Struktur unserer Disziplin ergeben hat. Dahinter liegt das Problem, dass die Wissensbestände der verschiedenen Teilbereiche der IB insgesamt zu selten konsequent miteinander verglichen und verbunden werden (vgl. Keukeleire/Schunz 2008). Dieses Problem verschärft sich noch dadurch, dass der Originalitätsdruck umso größer wird, je mehr Akteure ihren Platz in der Community suchen. Und je kreativer die Überlegungen der Beteiligten werden, desto größer wird nicht nur die Distanz zwischen den zu übersetzenden Bedeutungssystemen der verschiedenen Theorien, sondern auch der Aufwand, um Kommensurabilität und Kohärenz der Disziplin aufrecht zu erhalten.

\subsection{Ausmaß an Kooperation}

Eine weitere Dimension unbeabsichtigter Handlungsfolgen umfasst die (zu forcierende) Zusammenarbeit von Kollegen. Wie wenig Kooperation derzeit noch immer prämiert wird (abgesehen von der Bildung strategischer Allianzen im Kontext der Akquise von Drittmitteln vielleicht), geht insbesondere daraus hervor, dass von zwei oder mehr Autoren verfasste Publikationen weniger angesehen und akzeptiert sind als Arbeiten, für die Einzelne verantwortlich sind (vgl. Hackmann 2006: 111). Inspiriert von Peter M. Haas und Ernst B. Haas (2002: 576-577, Fn. 4) erweist sich ein Blick auf die Veröffentlichungspraxis einschlägiger Fachzeitschriften als durchaus instruktiv. So entfielen in den fünf Fünfjahreszeiträumen 1984-1988, 1989-1993, 1994-1998, 1999-2003 und 2004-2008 88,4\%, 78,6\%, 71,1\%, 59,6\% und $63,4 \%$ aller publizierten Beiträge in International Organization auf einzelne Autoren; in der erstmals 1994 erschienen Zeitschrift für Internationale Beziehungen

13 Für die Regel bestätigende gelungene Ausnahmen siehe Krell (1996), Jetschke/Liese (1998), Weller (2004) und Büger/Gadinger (2008). Während auch das Verfassen von Lehrbüchern (Krell (2000), Hellmann unter Mitarbeit von Baumann/Wagner (2006) oder Schieder/Spindler (2003)) mit zu wenig Anerkennung einhergeht, konzentrieren sich Bemühungen, den Überblick über das Fach zu wahren oder wiederherzustellen meist auf Handbücher - siehe Hellmann et al. (2003), Masala et al. (2010) oder für den angelsächsischen Sprachraum Carlsnaes et al. (2002), Reus-Smit/Snidal (2008) und nicht zuletzt das »Compendium Project« der ISA (2009). 
stammten in den drei Fünfjahreszeiträumen 1994-1998, 1999-2003 und 2004-2008 sogar $82,5 \%, 65,2 \%$ und 69,8\% aller veröffentlichten Beiträge von Einzelnen.

Eine Stärkung des Kooperationsgedankens gegenüber dem unsere Disziplin nach wie vor prägenden Veröffentlichungs-Individualismus hätte mindestens zwei positive Wirkungen. Erstens ließe die Beteiligung einer größeren Zahl von Forschern an einem Autorenkollektiv eine Steigerung der Qualität der einzelnen Beiträge erwarten, da die kritische Prüfung durch Kollegen so bereits während der Entstehung des Textes erfolgt. Zweitens aber würde die Zahl der publizierten und damit von den Mitgliedern der Community zu lesenden Beiträge reduziert, wenn wir den Autoren kollektiv verfasster Beiträge die gleichen Reputationsgewinne zusprechen würden, wie den Verfassern von Individualpublikationen. Dies wiederum würde neue Zeitkapazitäten schaffen, die für eine vertiefte und breitere Lektüre des Stands der Forschung anderer Theorien und Sektionen eingesetzt werden könnten.

Möglicherweise hinterfragen wir noch zu selten den offenbar tief verwurzelten Glauben daran, dass die großen (theoretischen) Würfe stets von einzelnen großen Geistern hervorgebracht werden. In der Vergangenheit mag sich dies aufgrund der gegebenen technischen und raum-zeitlichen Kommunikationsstrukturen in der Regel so ergeben haben. Unter globalisierten Bedingungen hingegen lassen sich gänzlich neue Kooperationsformen etablieren. Wir sollten daher prüfen, inwiefern unser Festhalten an Handlungsregeln, die letztlich an die Idee einer Individualkarriere zurückgebunden sind, zu einer Unterausschöpfung unseres kollektiv realisierbaren Potentials führt. Auch hier könnte sich das Ganze als größer erweisen als die Summe seiner Teile. ${ }^{14}$

\subsection{Sichtbarkeit und Diskussion von Forschungsergebnissen}

Ein drittes zentrales Problem unseres Faches besteht darin, dass die flächendeckende Initiierung von Forschungsprogrammen noch weitgehend aussteht, obwohl die Protagonisten der neuen Ansätze in den IB im Lauf der letzten zwanzig Jahre unermüdlich neue Theoriegebäude errichtet und dabei ganz erstaunliche Fortschritte erzielt haben. Mit anderen Worten: Obwohl die Irritationen im Zuge der neu entstandenen Teil-Teildisziplinen und der dadurch ermöglichte Theorienpluralismus durch realweltliche Entwicklungen ausgelöst worden sind, widmen wir der Auseinandersetzung mit theoretischen Gebilden - jenseits der notwendigen Schärfung unseres

14 Zur systematischen Rekonstruktion unerwünschter indirekter Folgen unseres kollektiven Handelns in den IB könnte auch gehören, das Problem des sogenannten »Matthäus-Effekts« besser bekannt zu machen und so zu dessen Lösung beizutragen. Als Matthäus-Effekt bezeichnete Robert K. Merton (1968: 58) in Bezug auf Mt 25,29 (»Denn wer da hat, dem wird gegeben werden, dass er die Fülle habe; wer aber nicht hat, von dem wird auch genommen, was er hat«) das Phänomen, dass ein angesehener Wissenschaftler für eine bestimmte wissenschaftliche Leistung mehr Ansehen gewinnt als ein weniger angesehener. Wie Stephen Cole (1972: 167) nachweisen konnte, stellt sich dieses Problem auch bei der Kooperation unterschiedlich angesehener Wissenschaftler, die gemeinsam publizieren. 
begrifflichen Instrumentariums - nach wie vor einen gehörigen Anteil unserer Kapazitäten. Diese Kapazitäten noch konsequenter zur Erforschung der politischen Wirklichkeit einzusetzen, wäre aber wohl schon allein deshalb wünschenswert, da die drängenden weltumspannenden Herausforderungen wie Armut, Hunger, Pandemien, Proliferation und Erderwärmung die Menschheit als Ganze betreffen und ihre systematische Erforschung von höchster Bedeutung ist. ${ }^{15}$ Denn während sich selbstbestimmte Forschung um Antworten auf (welt-)gesellschaftlich bedeutsame Fragen bemüht, erweckt das Innenleben unserer Disziplin mitunter den Anschein, als sei das Interesse an theoretischer Selbstverwirklichung - nicht selten flankiert von der Erfindung neuer, kaum anschlussfähiger Begrifflichkeiten - insgesamt größer als der Drang, den Lauf der Welt zu analysieren und der größeren Gesellschaft auf verständliche Art und Weise bekannt zu machen. Auch wenn dies der sympathischen epistemologischen Grundhaltung geschuldet sein mag, nach Falsifikationskriterien verfahrende Theorietests à la »King/Keohane/Verba« (1994) abzulehnen, wird die systematische Erforschung der politischen Wirklichkeit so zum akademischen Nebenschauplatz. Hochschild illustriert dies besonders plastisch:

$»[\ldots]$ the standard IR article consists of pushing a huge rock of theory up a steep hill, in order to roll it down to smash a few pebbles of fact at the bottom [...] « (Hochschild 2005: 11, zit. n. Katzenstein/Sil 2008: 110).

Diese Kritik am Theoretisieren als Selbstzweck soll nun nicht suggerieren, in den neuen Internationalen Beziehungen würde nicht geforscht. Wo immer neue Ansätze so weit entwickelt wurden, dass sie Forschung anzuleiten vermögen, sind, auch in Deutschland, Forschungsprogramme angelaufen (vgl. Deitelhoff/Wolf 2009: 462-467). Innerhalb der deutschen IB ist dies vornehmlich in den Sonderforschungsbereichen in Berlin (Governance in Räumen begrenzter Staatlichkeit) und Bremen (Staatlichkeit im Wandel) sowie im Frankfurter Exzellenzcluster Die Herausbildung normativer Ordnungen und am »Spitzenforschungsstandort« München der Fall.

Überraschenderweise kennzeichnet all diese »Leuchtturmprojekte« eine gewisse Zurückhaltung, was die sichtbare Präsentation der eigenen Forschungsergebnisse anbelangt. Denn so herausragend die Forschungsagenden und -fragen auch sein mögen, so entwicklungsfähig bleibt bislang doch die diesem Niveau entsprechende Struktur zur Präsentation und Diskussion der Forschungsergebnisse. Zwar werden die Publikationen sortiert nach den Namen der einzelnen Forscher im Internet aufgelistet, ${ }^{16}$ eine nach den verschiedenen Dimensionen der Forschungsagenda systematisierte Darstellung oder Diskussion der zentralen Befunde und deren Verknüpfung mit den Ergebnissen anderer Forschungsprogramme ist indes nicht ohne Weiteres erkennbar. Und dort, wo Ergebnisse in Form von Sammelbänden publiziert werden, sind sich die meisten Beiträge aufgrund ihrer gemeinsamen theoretischen

15 Damit ist nicht gesagt, Forschung sei auf ihre »politische Verwertbarkeit« auszurichten. Ebensowenig handelt es sich hier um ein verkapptes Plädoyer gegen Grundlagenforschung.

16 Vgl. hierzu SFB 597 (2009) und SFB 700 (2009). 
Prämissen und der fehlenden Heterogenität der Autoren meist zu ähnlich, um das Interesse einer größeren internationalen Leserschaft zu wecken. Obwohl Deitelhoff/ Wolf (2009: 458) also mit Recht von einer »zunehmend auch konzertierten Bearbeitung vieler wichtiger Untersuchungsfelder« in der deutschen IB sprechen, bleiben die Ergebnisse dieser Bemühungen zu oft unsichtbar.

Dass empirischer Forschung in unserer Disziplin nur eine Nebenrolle zukommt, verdeutlicht auch der Aspekt des Veröffentlichens von Forschungsergebnissen in Fachzeitschriften. Diese Praxis ist längst noch nicht zur Regel geworden und wäre deshalb gezielt zu fördern; schließlich ist die »institutionelle Vorstellung von Wissenschaft als Teil der öffentlichen Sphäre« ja mit »dem Zwang zur Kommunikation von Resultaten verknüpft« (Merton 1972: 51). Weingart (1972: 16-17) merkte dazu schon vor vier Jahrzehnten an:

»Die Produktionsexplosion in den Wissenschaften führt zur zumindest potentiellen Auflösung herkömmlicher Kommunikationsweisen wie z.B. der Veröffentlichung von Ergebnissen in Fachzeitschriften [...] Die Diffusion wissenschaftlicher Ergebnisse und ihrer Kontrolle durch die akademische Öffentlichkeit wird aufgrund dessen zu den wohl schwerstwiegenden Strukturproblemen der Wissenschaft in den kommenden Jahren«.

Weiterhin werden die verschiedenen Befunde, die zu einem Problem vorliegen, nur selten zeitnah miteinander diskutiert. Dies ist gerade deswegen eine höchst unerwünschte Folge der gegenwärtigen disziplininternen Handlungsstruktur, weil so den Forschern nach getaner Forschungsarbeit die »Gegenleistung« der Community in Form einer kompetenten Beurteilung der Ergebnisse meist versagt bleibt. Trotz des massiv angestiegenen und inzwischen enorm ausdifferenzierten Theoriebestandes wird der größte Teil der uns kollektiv zur Verfügung stehenden Energie also noch immer in Umbaumaßnahmen der Theoriegebäude investiert. Darüber droht in Vergessenheit zu geraten, dass die Frage nach der erklärungskräftigsten Theorie ohnehin niemals abgeschlossen werden kann. Früher oder später muss die Theoriearbeit somit in den Dienst empirischer Analysen gestellt werden - und dieser Schritt ist in einer konsequenten Form noch immer nicht zur Gänze vollzogen. ${ }^{17}$ Mit Blick auf die deutsche IB stellen Deitelhoff/Wolf (2009: 468) gar fest, dass neue Theorien »als >latest fashion « zwar gern importiert und ausprobiert, aber schon weniger gern zu Ende gedacht oder gar mühsam und empirisch erschöpfend abgearbeitet « werden.

Verschärft wird die Problematik unzureichender Sichtbarkeit und Diskussion von Forschungsergebnissen noch dadurch, dass zumindest in den deutschen IB noch immer kein einschlägiger Ort zur Publikation von Methodenbeiträgen identifizierbar ist. Dies kann im Grunde nur bedeuten, dass der praktische Umgang mit Methoden im Rahmen von Forschung nur einen Bruchteil unserer Arbeitsrealität aus-

17 Auch der berechtigte Hinweis darauf, dass $»[t]$ here is nothing as practical as a good theory« (Lewin 1951: 161), der ja vor allem verdeutlicht, dass jede Form von Weltbetrachtung immer schon zugleich theoretisch angeleitet ist und theoriegenerierend wirkt, kann doch die Unterschiede bezüglich der hervorgebrachten praktischen Wirkungen nicht einebnen, die aus der Beschäftigung mit eher formaltheoretischen Fragen der Expertenrealität einerseits und der stärker lebensweltlich bezogenen Erforschung der sozio-politischen Realität der größeren (national oder global definierten) Gesellschaft andererseits resultieren. 
macht. Hätte sich unsere Disziplin bereits konsequenter der Forschungspraxis zugewendet, dann wäre diese strukturelle Lücke wohl schon viel häufiger bemerkt, Lösungsmöglichkeiten wären diskutiert und Abhilfe wäre geschaffen worden. Es mag sein, dass ein solcher Ort, an dem systematisch Fragen von Methodologie und Methode behandelt werden könnten, in dem Ruf steht, lediglich als Austragungsort für epistemologische »Stellvertreterkriege« herhalten $\mathrm{zu}$ müssen. Wo Forschung von einer Hinwendung zum Gegenstand gekennzeichnet ist, sind solche Sorgen jedoch unbegründet. Hier sind Methoden ihres Fetischcharakters (vgl. Adorno 1972) beraubt und dienen als bloße Werkzeuge zur Bearbeitung dessen, was die internationalen Beziehungen (alt wie neu) den Forschern an konkreten Gegenständen beschert haben.

\section{Resümee}

Der realweltliche Wandel nach dem Ende des Ost-West-Konflikts ist auch für das Innenleben der politikwissenschaftlichen Teildisziplin der Internationalen Beziehungen nicht ohne Folgen geblieben. Die Theorien und Forschungsansätze der $a l$ ten IB wurden um neue Strömungen ergänzt; neue Möglichkeiten und neue Herausforderungen haben sich ergeben. Eine dieser Herausforderungen besteht darin, den disziplininternen Bestand an Handlungsroutinen daraufhin zu untersuchen, welche unerwünschten indirekten Folgen er zeitigt. Der vorliegende Beitrag hat hierzu einen ersten Versuch unternommen. Bei der Selbsterforschung der Disziplin handelt es sich jedoch um eine kollektive Aufgabe, die institutionalisiert erfolgen sollte. Daher hoffen wir auf weitere Arbeiten, die nach unerwünschten indirekten Folgen unseres disziplininternen Handelns fragen und Vorschläge $\mathrm{zu}$ deren Regulierung formulieren.

In den Vordergrund gerückt haben wir hier die unerwünschten Handlungsfolgen, deren Regulierung uns am vordringlichsten erscheint. Wir haben zunächst argumentiert, dass es der Aufrechterhaltung der Kohärenz des Faches eher abträglich ist, die Prämierung von Überblicksarbeiten der Anerkennung innovativer Theoriebeiträge deutlich nachzuordnen. Sodann verwiesen wir darauf, dass der ausgeprägte Veröffentlichungs-Individualismus nicht nur wertvolle Zeitressourcen bindet, sondern auch die Kooperationsneigung der Forscher schwächt. Schließlich haben wir angemerkt, dass das Fehlen einer Kultur der systematischen Auseinandersetzung mit den Ergebnissen bestehender Forschungsprogramme zur Folge hat, dass die Entwicklung theoretischer Annahmen und Modelle weitaus mehr Prestige bringt als das geduldige Erforschen der Welt

Zur Regulierung dieser indirekten unerwünschten Handlungsfolgen wurden alternative Handlungsregeln vorgeschlagen. Auf mittlere Sicht mögen sie bewirken: 
- $\quad$ eine Zunahme der Kohärenz des Wissensbestandes der IB;

- $\quad$ einen Anstieg der Kooperation, insbesondere bei der Produktion von Publikationen;

- sowie eine sichtbarere und intensivere Diskussion von Forschungsergebnissen und deren Zustandekommen.

Zusammengenommen bräuchte auf diese Weise deutlich weniger Zeit für den aufwändigen Ausbau einzelner theoretischer Großprojekte reserviert zu werden. Stattdessen würden immer mehr AnhängerInnen der verschiedenen Theorien dazu ermutigt, die sich fortlaufend verändernden weltpolitischen Realitäten zu erkunden und dabei die verschiedenen und je für sich äußerst wertvollen Wissenskulturen kreativ miteinander zu verbinden.

\section{Literatur}

Adorno, Theodor W. 1972: Soziologie und empirische Forschung, in: Adorno, Theodor W.: Gesammelte Schriften, Bd. 8: Soziologische Schriften I, Frankfurt a. M., 196-215.

Albert, Mathias 2003: Entgrenzung und internationale Beziehungen: Der doppelte Strukturwandel eines Gegenstandes und seines Faches, in: Hellmann et al. 2003, 555-576.

Bartelson, Jens 2000: Three Concepts of Globalization, in: International Sociology 15: 2, 180-196.

Bauer, Harry/Brighi, Elisabetta 2009: Conclusions: On the Obstacles and Promises of Pragmatism in International Relations, in: Bauer, Harry/Brighi, Elisabetta (Hrsg.): Pragmatism in International Relations, London, 163-170.

Büger, Christian/Gadinger, Frank 2007: Reassembling and Dissecting: International Relations Practice from a Science Studies Perspective, in: International Studies Perspectives 8: $1,90-110$.

Büger, Christian/Gadinger, Frank 2008: Praktisch gedacht! Praxistheoretischer Konstruktivismus in den Internationalen Beziehungen, in: Zeitschrift für Internationale Beziehungen 15: 2, 273-302.

Bull, Hedley 1977: The Anarchical Society: A Study of Order in World Politics, London.

Carlsnaes, Walter 2002: Foreign Policy, in: Carlsnaes et al. 2002, 331-349.

Carlsnaes, Walter/Risse, Thomas/Simmons, Beth A. (Hrsg.) 2002: Handbook of International Relations, London.

Cerny, Philip G. 1995: Globalization and the Logic of Collective Action, in: International Organization 49: 4, 595-625.

Cerny, Philip G. 1997: Paradoxes of the Competition State: The Dynamics of Political Globalization, in: Government and Opposition 32: 2, 251-274.

Checkel, Jeffrey 2001: Why Comply? Social Learning and European Identity Change, in: International Organization 55: 3, 553-588.

Cole, Stephen 1972: Wissenschaftliches Ansehen und die Anerkennung wissenschaftlicher Leistungen, in: Weingart, Peter (Hrsg.): Wissenschaftssoziologie I: Wissenschaftliche Entwicklung als sozialer Prozeß, Frankfurt a. M., 165-187.

Crawford, Beverly 2007: Power and German Foreign Policy, New York, NY.

Deitelhoff, Nicole/Wolf, Klaus Dieter 2009: Der Widerspenstigen Selbst-Zähmung? Zur Professionalisierung der Internationalen Beziehungen in Deutschland, in: Politische Vierteljahresschrift 50: 3, 451-475.

Dewey, John 2001: Die Öffentlichkeit und ihre Probleme, Berlin.

Dewey, John 2004: Die Notwendigkeit einer Selbsterneuerung der Philosophie, in: ders.: Erfahrung, Erkenntnis und Wert, Frankfurt a. M., 145-195. 
Dewey, John 2008: Logik. Die Theorie der Forschung, Frankfurt a. M.

Finnemore, Martha/Sikkink, Kathryn 1998: International Norm Dynamics and Political Change, in: International Organization 52: 4, 887-917.

Friedrichs, Jörg/Kratochwil, Friedrich 2009: On Acting and Knowing: How Pragmatism can advance International Relations Research and Methodology, in: International Organization 63: 4, 701-731.

Griffiths, Martin/O'Callaghan, Terry 2001: »The End of International Relations?«, in: Crawford, Robert/Jarvis, Darryl (Hrsg.): International Relations: Still an American Social Science: Towards Diversity in International Thought?, Albany, NY, 187-201.

Haas, Peter M./Haas, Ernst B. 2002: Pragmatic Constructivism and the Study of International Institutions, in: Millennium. Journal of International Studies 31: 3, 573-601.

Hackmann, Kristina 2006: Professorinnen in der Mathematik. Karrierewege und disziplinäre Verortungen, in: Ebeling, Smilla/Schmitz, Sigrid (Hrsg.): Geschlechterforschung und Naturwissenschaften, Wiesbaden, 95-116.

Hellmann, Gunther 2002: Creative Intelligence: Pragmatism as a Theory of Thought and Action (Paper prepared for presentation at the »Millennium« Special Issue Conference on »Pragmatism in International Relations Theory«, London 12.10.2002).

Hellmann, Gunther 2010: Pragmatismus, in: Masala et al. 2010, 148-181.

Hellmann, Gunther/Wolf, Klaus Dieter/Zürn, Michael (Hrsg.) 2003: Die neuen Internationalen Beziehungen. Forschungsstand und Perspektiven in Deutschland, Baden-Baden.

Hellmann, Gunther unter Mitarbeit von Baumann, Rainer/Wagner, Wolfgang 2006: Deutsche Außenpolitik. Eine Einführung, Wiesbaden.

Humrich, Christoph 2006: Germany, in: Jørgensen, Knud E./Knudsen, Tonny B. (Hrsg.): International Relations in Europe. Traditions, Perspectives and Destinations, London, 72-99.

ISA 2009: International Studies Association Compendium Project, in: http://www.isanet.org/ compendium; 25.04.2012.

ISA 2011: International Studies Association Website, in: http://www.isanet.org/groups/isa-sections.html; 25.04.2012.

Jetschke, Anja/Liese, Andrea 1998: Kultur im Aufwind. Zur Rolle von Bedeutungen, Werten und Handlungsrepertoires in den internationalen Beziehungen, in: Zeitschrift für Internationale Beziehungen 5: 1, 149-179.

Joerges, Christian 2001: Transnationale »deliberative Demokratie« oder »deliberativer Supranationalismus «? Anmerkungen zur Konzeptualisierung legitimen Regierens jenseits des Nationalstaats bei Rainer Schmalz-Bruns, in: Zeitschrift für Internationale Beziehungen 7: 2, 145-161.

Joerges, Christian 2006: Freier Handel mit riskanten Produkten? Die Erosion nationalstaatlichen und die Emergenz transnationalen Regierens, in: Leibfried, Stephan/Zürn, Michael (Hrsg.): Transformation des Staates? Frankfurt a. M., 151-193.

Karp, Regina 2009: Germany. A »Normal« Global Actor?, in: German Politics 18: 1, 12-35.

Katzenstein, Peter J./Sil, Rudra 2008: Eclectic Theorizing in the Study and Practice of International Relations, in: Reus-Smit/Snidal 2008, 109-130.

Keck, Margaret E./Sikkink, Kathryn 1998: Activists beyond Borders: Advocacy Networks in International Politics, Ithaca, NY.

Keohane, Robert O. 2008: Big Questions in the Study of World Politics, in: Reus-Smit/Snidal 2008, 708-715.

Keukeleire, Stephan/Schunz, Simon 2008: Foreign Policy, Globalization and Global Governance: The European Union's Structural Foreign Policy (Paper prepared for the ECPR Standing Group on the European Union, Fourth Pan-European Conference on EU Politics, Riga 25. bis 28.09.2008).

King, Gary/Keohane, Robert O./Verba, Sidney 1994: Designing Social Inquiry: Scientific Inference in Qualitative Research, Princeton. 
Knorr-Cetina, Karin 1984: Die Fabrikation von Erkenntnis. Zur Anthropologie der Naturwissenschaft, Frankfurt a. M.

Kratochwil, Friedrich 2009: Ten Points to Ponder About Pragmatism: Some Critical Reflections on Knowledge Generation in the Social Sciences, in: Bauer, Harry/Brighi, Elisabetta (Hrsg.): Pragmatism in International Relations, London, 11-25.

Krell, Gert 1996: Feminismus und Internationale Beziehungen. Zwischen Dekonstruktion und Essentialisierung, in: Zeitschrift für Internationale Beziehungen 3: 1, 149-181.

Krell, Gert 2000: Weltbilder und Weltordnung. Einführung in die Theorie der internationalen Beziehungen, Baden-Baden.

Kuhn, Thomas S. 1976: Die Struktur wissenschaftlicher Revolutionen, 2. Auflage, Frankfurt a. M.

Lakatos, Imre 1970: Falsification and the Methodology of Scientific Research Programmes, in: Lakatos, Imre/Musgrave, Alan (Hrsg.): Criticism and the Growth of Knowledge, Cambridge, 128-200.

Lapid, Yosef 1989: The Third Debate: On the Prospects of International Theory in a Post-Positivist Era, in: International Studies Quarterly 33: 3, 235-254.

Latour, Bruno 2000: Die Hoffnung der Pandora. Untersuchungen zur Wirklichkeit der Wissenschaft, Frankfurt a. M.

Leibfried, Stephan/Zürn, Michael 2006: Von der nationalen zur post-nationalen Konstellation, in: dies. (Hrsg.): Transformation des Staates? Frankfurt a. M., 19-65.

Lewin, Kurt 1951: Field Theory in Social Science: Selected Theoretical Papers, New York, NY.

Luke, Timothy W. 1993: Discourses of Disintegration, Texts of Transformation: Re-Reading Realism in the New World Order, in: Alternatives 18: 2, 229-258.

Masala, Carlo/Sauer, Frank/Wilhelm, Andreas (Hrsg.) 2010: Handbuch der Internationalen Politik, Wiesbaden.

Merton, Robert K. 1968: The Matthew Effect in Science, in: Science 159: 3810, 56-63.

Merton, Robert K. 1972: Wissenschaft und demokratische Sozialstruktur, in: Weingart, Peter (Hrsg.): Wissenschaftssoziologie I: Wissenschaftliche Entwicklung als sozialer Prozeß, Frankfurt a. M., 45-59.

Peirce, Charles S. 1992: How to Make Our Ideas Clear, in: Houser, Nathan/Kloesel, Christian (Hrsg.): The Essential Peirce, Selected Philosophical Writings, Bd. 1, Bloomington, IN, 124-141.

Reus-Smit, Christian/Snidal, Duncan (Hrsg.) 2008: The Oxford Handbook of International Relations, Oxford.

Risse, Thomas 2003: Konstruktivismus, Rationalismus und Theorien Internationaler Beziehungen. Warum empirisch nichts so heiß gegessen wird, wie es theoretisch gekocht wurde, in: Hellmann et al. 2003, 99-132.

Risse, Thomas/Ropp, Stephen C./Sikkink, Kathryn (Hrsg.) 1999: The Power of Human Rights: International Norms and Domestic Change, Cambridge.

Rittberger, Volker/Hummel, Hartwig 1990: Die Disziplin »Internationale Beziehungen« im deutschsprachigen Raum auf der Suche nach ihrer Identität. Entwicklung und Perspektiven, in: Rittberger, Volker (Hrsg.): Theorien der Internationalen Beziehungen. Bestandsaufnahme und Forschungsperspektiven, Opladen (PVS-Sonderheft 21), 17-47.

Rosenau, James N. 1999: Toward an Ontology for Global Governance, in: Hewson, Martin/ Sinclair, Timothy J. (Hrsg.): Approaches to Global Governance Theory, Albany, NY, 287-302.

Rosenau, James N./Czempiel, Ernst-Otto (Hrsg.) 1992: Governance Without Government: Order and Change in World Politics, Cambridge.

Ruggie, John G. 2004: Reconstituting the Global Public Domain: Issues, Actors, and Practices, in: European Journal of International Relations 10: 4, 499-531.

Sassen, Saskia 1996: Losing Control? Sovereignty in an Age of Globalization, New York, NY. 
Schieder, Siegfried/Spindler, Manuela 2003: Theorien der Internationalen Beziehungen, Opladen.

Sending, Ole J./Neumann, Iver B. 2006: Governance to Governmentality. Analyzing NGOs, States, and Power, in: International Studies Quarterly 50: 3, 651-672.

SFB 597 2009: Aktuelle Publikationen des Sonderforschungsbereichs 597, Staatlichkeit im Wandel, in: http://www.sfb597.uni-bremen.de/pages/publikationen.php?SPRACHE=de; 25.04.2012.

SFB 700 2009: Publikationen, Sonderforschungsbereich 700, Governance in Räumen begrenzter Staatlichkeit, in: http://www.sfb-governance.de/publikationen/index.html; 25.04.2012.

Smith, Steve/Hadfield, Amelia/Dunne, Timothy 2008: Introduction, in: dies. (Hrsg.): Foreign Policy. Theories, Actors, Cases, Oxford, 1-8.

Tickner, Arlene B./Wcever, Ole 2009: International Relations Scholarship around the World, London.

Waltz, Kenneth N. 1979: Theory of International Politics, New York, NY.

Waever, Ole 1998: The Sociology of a Not So International Discipline: American and European Developments in International Relations, in: International Organization 52: 4, 687-727.

Weingart, Peter 1972: Die Wissenschaftsforschung und wissenschaftssoziologische Analyse, in: ders. (Hrsg.): Wissenschaftssoziologie I: Wissenschaftliche Entwicklung als sozialer Prozeß, Frankfurt a. M., 11-42.

Weller, Christoph 2004: Kenntnisse, Einsichten und Perspektiven der Internationalen Beziehungen. Forschung und Lehre, neuester Stand, in: Politische Vierteljahresschrift 45: 2, 263-276.

Wendt, Alexander 1999: Social Theory of International Politics, Cambridge.

Wendt, Alexander 2001: What is International Relations For? Notes Toward a Postcritical View, in: Jones, Richard W. (Hrsg.): Critical Theory and World Politics, Boulder, CO, 205-224.

WISC 2009: World International Studies Committee Website, in: http://www.wiscnetwork.org; 25.04.2012.

Wolf, Klaus Dieter/Hellman, Gunther 2003: Die Zukunft der Internationalen Beziehungen in Deutschland, in: Hellmann et al. 2003, 577-603.

Zangl, Bernhard/Zürn, Michael 2003: Frieden und Krieg. Sicherheit in der nationalen und post-nationalen Konstellation, Frankfurt a. M.

Zürn, Michael 2005: Regieren jenseits des Nationalstaates. Globalisierung und Denationalisierung als Chance, Frankfurt a. M. 